\title{
Understanding and Controlling the 1,4-Phenylene Diisocyanide- Gold Oligomer Formation Pathways
}

\author{
Michael Garvey, ${ }^{\dagger}$ John Kestell, ${ }^{\dagger}$ Rasha Abuflaha, $^{\dagger}$ Dennis W. Bennett, ${ }^{\dagger}$ Graeme Henkelman, ${ }^{\dagger}$ \\ and Wilfred T. Tysoe*, $\dagger$ \\ ${ }^{\dagger}$ Department of Chemistry and Biochemistry and Laboratory for Surface Studies, University of Wisconsin-Milwaukee, Milwaukee, \\ Wisconsin 53211, United States \\ ${ }^{\ddagger}$ Department of Chemistry, The University of Texas at Austin, Austin, Texas 78712-0165, United States
}

\section{Supporting Information}

ABSTRACT: The pathways for the spontaneous self-assembly of one-dimensional oligomeric chains from the adsorption of 1,4-phenylene diisocyanide (PDI) on $\mathrm{Au}(111)$ surface are explored using density functional theory. It has been shown previously that the chain comprises repeating - $(\mathrm{Au}-\mathrm{PDI})$ - structures. The results show that the chains form from mobile $\mathrm{Au}-\mathrm{PDI}$ adatom complexes and that chains propagate by the adatom complex coupling to a terminal isocyanide group which lies close to parallel to the surface and the activation barrier for this propagation step is $\sim 28 \mathrm{~kJ} / \mathrm{mol}$. It is also found that the Au-PDI adatom complex is attracted to the terminal isocyanide, thereby facilitating the oligomerization process. The insights into the oligomerization pathway are used to explore whether an external electric field applied to diisocyanide functionalized molecules that contain a dipole moment can be used to align them. It is found that molecules with dipole moments of $\sim 1 \mathrm{D}$ show significant alignment with an electric field of $\sim 10^{8} \mathrm{~V} / \mathrm{m}$ and almost complete alignment when the electric field reaches $\sim 10^{9} \mathrm{~V} / \mathrm{m}$. This suggests that the selfassembly chemistry of dipolar diisocyanides can be used to create oriented systems.

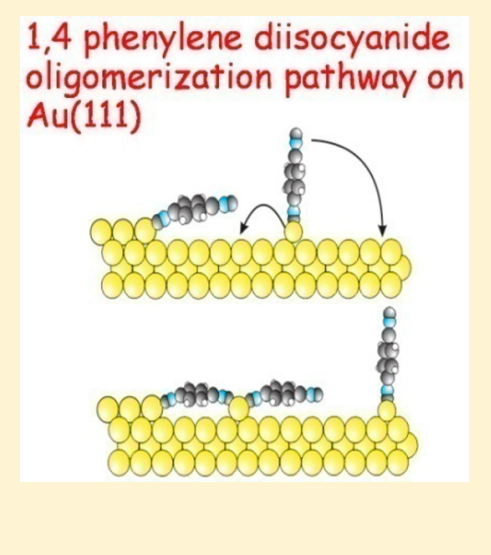

\section{INTRODUCTION}

1,4-Phenylene diisocyanide (PDI) self-assembles on an $\mathrm{Au}(111)$ surface at room temperature resulting in the formation of one-dimensional chains that comprise alternating gold and PDI units. ${ }^{1-3}$ It has been shown that they form by extracting gold atoms from low-coordination sites on the gold substrate and adatom structures are relatively common on coinage metals. $^{4-7}$ Density functional theory (DFT) calculations indicate that the formation of such one-dimensional oligomers is energetically favorable, and the resulting structure is displayed in Figure 1. The nature of the one-dimensional chain has been confirmed using reflection-absorption infrared spectroscopy (RAIRS) and by comparing the simulated scanning tunneling microscopy (STM) images calculated for the structure shown in Figure 1 with experiment, where the two show good agreement. ${ }^{8}$ In addition to forming on gold single crystal substrates, it has been found that the -(Au-PDI)oligomers are capable of bridging between gold nanoparticles deposited onto an insulating mica substrate, thereby providing a conductive pathway between the nanoparticles. ${ }^{9}$ These results suggest the possibility of using such self-assembly chemistry as a basis for fabricating molecular electronic architectures. However, being able to accomplish this would necessitate being able to control the oligomer structure. For example, Aviram and Ratner suggested that molecular diodes could be fabricated from aligned molecules containing electronically separated electron donor and acceptor units. ${ }^{10}$ Some experimental evidence has been provided that this might be possible using break-junction experiments. ${ }^{11-13}$

Since 1,4-phenylene diisocyanide reacts relatively rapidly to form oligomeric species on $\mathrm{Au}(111)$, the formation time scales are too short to allow the formation pathway to be interrogated experimentally. In the following, we have therefore used DFT calculations to propose plausible, energetically favorable oligomerization pathways and to use these insights to propose possible strategies for controlling the structure and orientation of the surface molecular units.

\section{THEORETICAL METHODS}

Density functional theory calculations were performed with the projector augmented wave (PAW) method $^{14}$ as implemented in the Vienna ab initio simulation package, VASP. ${ }^{15}$ The exchange-correlation potential was described using the generalized gradient approximation (GGA) of Perdew, Burke and Ernzerhof. ${ }^{16}$ A cutoff of $400 \mathrm{eV}$ was used for the planewave basis set, and the wave functions and electron density were converged to within $1 \times 10^{-5} \mathrm{eV}$. The first Brillouin zone was sampled with a $4 \times 4 \times 1 \Gamma$-centered k-point mesh. Geometric relaxations were considered to be converged when the force was less than $0.01 \mathrm{eV} / \AA$ on all unrestricted atoms. Since van der Waals forces have been found to provide a large

\footnotetext{
Received: May 15, 2014

Revised: August 20, 2014

Published: August 20, 2014
} 
(a)

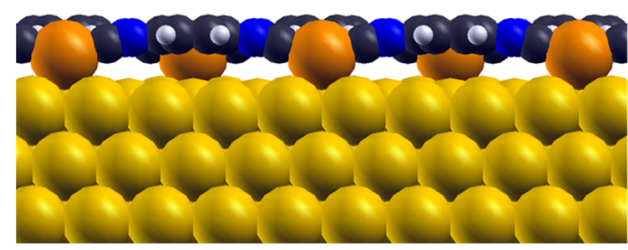

(b)

(c)

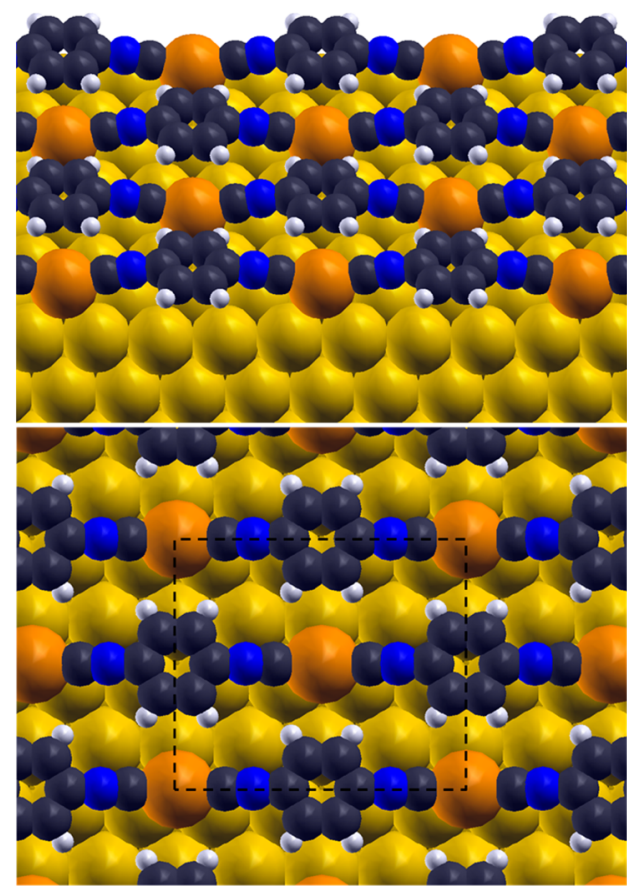

Figure 1. Structure of $(\mathrm{Au}-\mathrm{PDI})$ oligomers on gold calculated using DFT shown (a) a side view, (b) an angled view, and (c) a top views of the oligomer chains.

contribution to the binding energies on coinage metals surfaces, ${ }^{17-22}$ calculations were also performed by including van der Waals corrections using the method of Tkatchenko and Scheffler (TS). ${ }^{23}$ Nudged elastic band (NEB) calculations were performed using the climbing image method. ${ }^{24}$

\section{RESULTS AND DISCUSSION}

The results of DFT calculations of PDI-Au oligomer chains are presented for reference. The surface structure was constructed by bridging gold adatoms placed on a $\mathrm{Au}(111)$ substrate aligned along the close-packed $\langle 1 \overline{1} 0\rangle$ directions, corresponding to the orientations on the linear chains observed by STM on $\mathrm{Au}(111) .{ }^{1-3}$ The gold adatoms were separated by foursubstrate lattice-translation distances, corresponding to the experimental repeat distance of $\sim 1.1 \mathrm{~nm}$. The PDI molecule was then placed between the gold adatoms and the structure allowed to relax. The resulting structures are displayed in Figure 1, where Figure 1a shows a side view of the Au-PDI-Au bridging units, Figure $1 \mathrm{c}$ illustrates a top view, and Figure $1 \mathrm{~b}$ an angle view. The isocyanide groups are angled slightly $\left(\right.$ at $\sim 13^{\circ}$ ) with respect to the surface, and the molecule has local $C_{2 v}$ symmetry, with the aryl ring oriented parallel to the $\mathrm{Au}(111)$ plane. The binding energy, calculated from the difference between the energy of the final structure shown in Figure 1, and the energies of the gold-adatom-covered surface plus the energy of gas-phase PDI is ca. $-177 \mathrm{~kJ} / \mathrm{mol}$ per molecular unit.

Since the oligomer consists of repeating $\mathrm{Au}-\mathrm{PDI}$ units, it is proposed to result from a $\mathrm{Au}-\mathrm{PDI}$ adatom complex that forms by extracting gold atoms from low-coordination sites on the surface. To explore the structure and mobility of this putative precursor, a PDI molecule was placed onto a gold adatom on a $\mathrm{Au}(111)$ surface and the geometry optimized. It was found that the most stable site for the gold adatom in the complex was at a face-centered cubic (fcc), 3-fold hollow site. The structure is depicted in Figure 2b. Similar structures for the Au-PDI

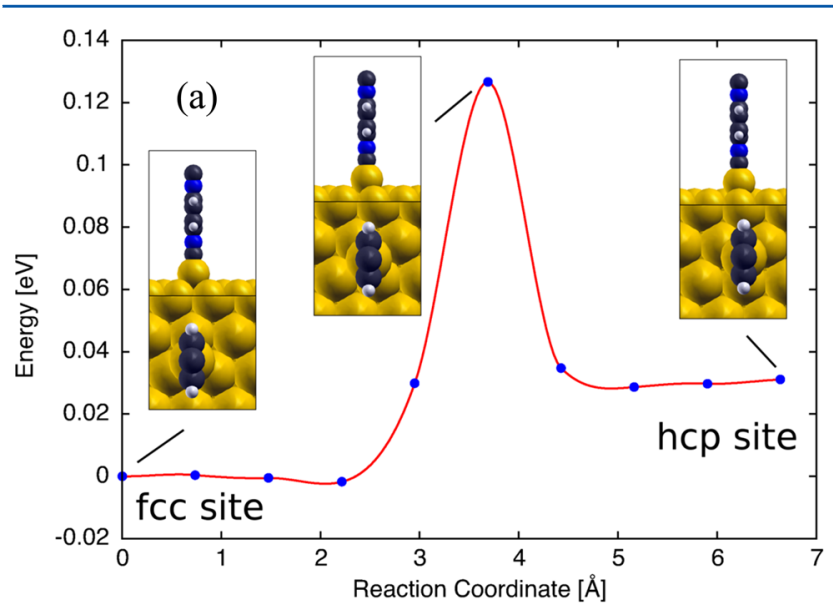

(b)

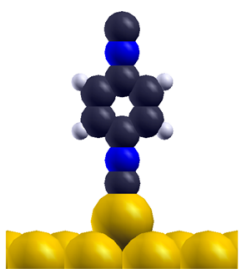

Figure 2. (a) The calculated energy profile for diffusion of the adatom complex from the most stable, fcc 3 -fold hollow site to the adjacent hcp site. (b) The structure of the most stable, vertical Au-PDI adatom complex on a $\mathrm{Au}(111)$ substrate.

adatom complex on gold have been found previously. ${ }^{25}$ The binding energy of the PDI to the gold adatom was calculated from the difference between the energy of the isocyanide molecule bound to a gold adatom on the substrate and the energies of an isolated, gas-phase PDI molecule and a $\mathrm{Au}(111)$ surface with a gold adatom yielding an energy of $-1.42 \mathrm{eV}$ $(\sim 137 \mathrm{~kJ} / \mathrm{mol})$. The structure of the Au-PDI adatom complex was recalculated by including van der Waals interactions, which increased the energy to $-1.58 \mathrm{eV}$ ( $\mathrm{ca} .-152 \mathrm{~kJ} / \mathrm{mol}$ ) without any discernible change in geometry. While the most stable structure was observed with the principal axis of the PDI molecule perpendicular to the surface, the energy to tilt the molecule is fairly small. ${ }^{25,26}$ This is discussed in greater detail below. Since oligomerization of this adatom complex necessitates it being relatively mobile on the surface, the energy for it to diffuse from the most stable fcc, 3-fold hollow site to the closest (hexagonal-close packed (hcp)) 3-fold-hollow site was calculated. The calculated plot of energy versus reaction coordinate is displayed in Figure $2 \mathrm{~b}$ along with selected structures along the reaction pathway. An animation of the motion is available in the Supporting Information. The energy of the fcc site is almost degenerate with the hcp site, which is slightly less stable, and the barrier for diffusion is relatively low at $\sim 0.13 \mathrm{eV}(\sim 13 \mathrm{~kJ} / \mathrm{mol})$. The time for the adatom complex to move to an adjacent site can be estimated by using a standard pre-exponential factor of $2 \times 10^{12} \mathrm{~s}^{-1}$ to be 

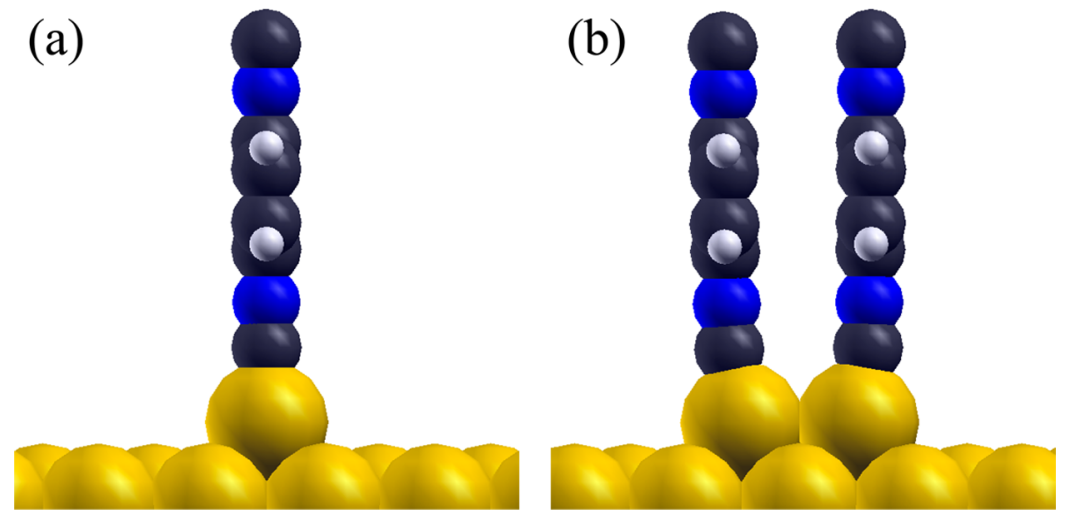

Figure 3. Side views of the calculated structure of (a) one vertical Au-PDI adatom complex and (b) two adjacent Au-PDI adatom complexes on a $\operatorname{Au}(111)$ substrate.

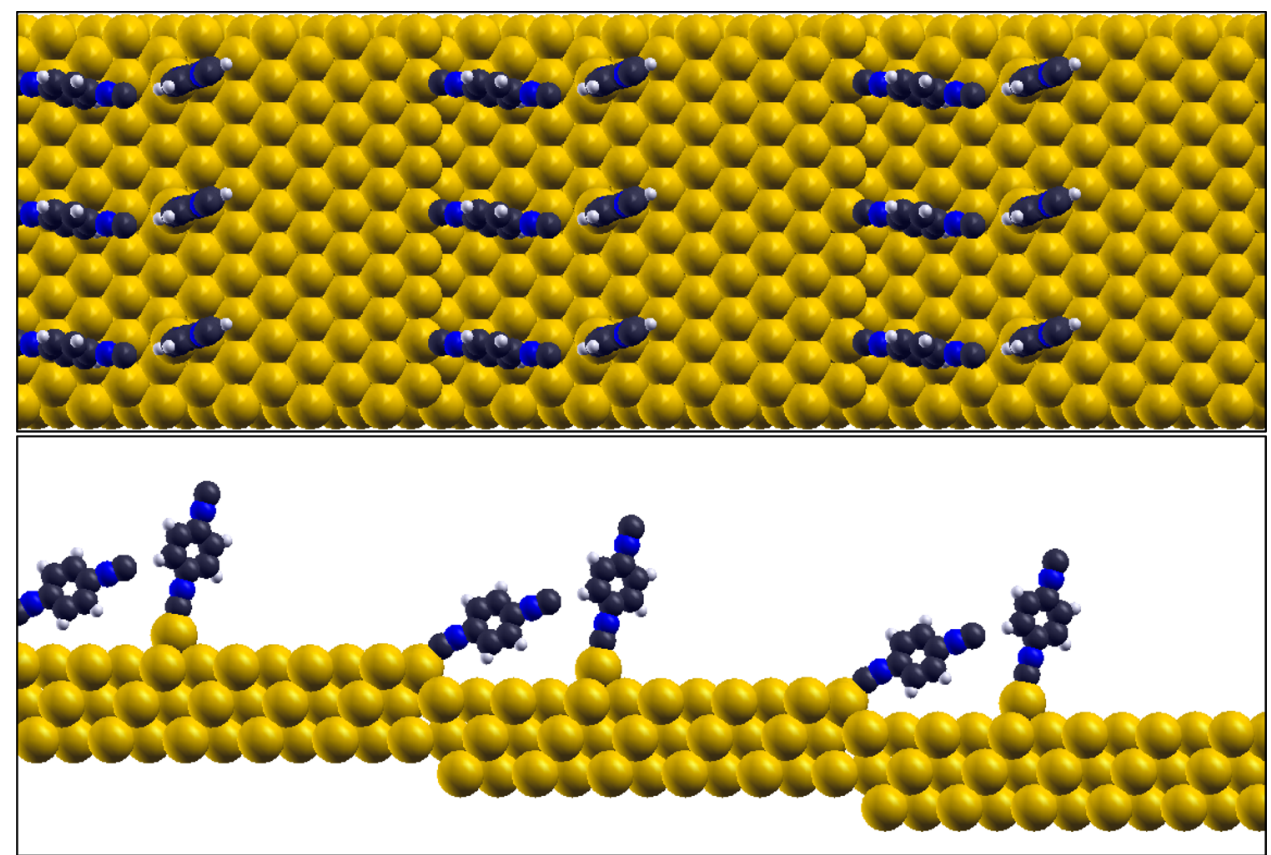

Figure 4. Calculated structure of a PDI molecule bound to a gold step, with a second PDI molecule placed close to it as an initial configuration for calculating the reaction barrier.

$\sim 2 \times 10^{-10} \mathrm{~s}$ at $300 \mathrm{~K}$ so that the adatom complex is mobile on the surface.

It is possible that these mobile, upright $\mathrm{Au}-\mathrm{PDI}$ complexes could agglomerate due to attractive $\pi-\pi$ interactions between them, which would inhibit $\mathrm{Au}-\mathrm{PDI}$ chain growth. Therefore, two $\mathrm{Au}$-PDI complexes were placed at adjacent 3-fold hollow sites and allowed to relax where the calculations were performed by including van der Waals' interactions. A side view of the final geometry is shown in Figure $3 \mathrm{~b}$ compared with the geometry of the isolated adatom complex in Figure 3a. The binding energy per Au-PDI complex for the adjacent configuration is $\sim 10 \mathrm{~kJ} / \mathrm{mol}$ less stable than for an isolated complex, which means it would cost $\sim 20 \mathrm{~kJ} / \mathrm{mol}$ to form from two isolated complexes. Therefore, agglomeration of the vertical Au-PDI complexes would not be expected to occur.

There are two possible ways in which the mobile adatom complex could oligomerize. In the first, the Au-PDI adatom complex could diffuse to a step edge or a gold adatom at the terminus of a growing - (Au-PDI $)$ - oligomer chain, and tilt to coordinate the free isocyanide group to the gold terminus. This process creates a new terminus for subsequent oligomerization steps. The relatively low energies required to tilt the PDI on the adatom complex suggests that this should be energetically feasible. ${ }^{25}$ However, when the gold-adatom complex is placed adjacent to a gold atom terminating the chain, the PDI molecule merely tilts and does not form a bond between the isocyanide group and the gold atom. Presumably this might be possible if the adatom complex tilted when located on a site an appropriate distance for the chain terminus $(\sim 4$ lattice spacings), but the mobility of the adatom complex calculated above suggests that the residence time at that site would be too short for this step to occur with a high probability. We therefore explored the alternative possibility in which an isocyanide-terminated chain couples directly with the gold atom in the gold adatom complex. This was initially explored for a PDI molecule bound to a step edge, to mimic the presence of a low-coordination site on the surface, with the structure shown in Figure 4. It is anticipated that a similar processes will occur at other low-coordination sites such as at "elbow" dislocation sites of the $\mathrm{Au}(111)$ reconstruction. This reveals 


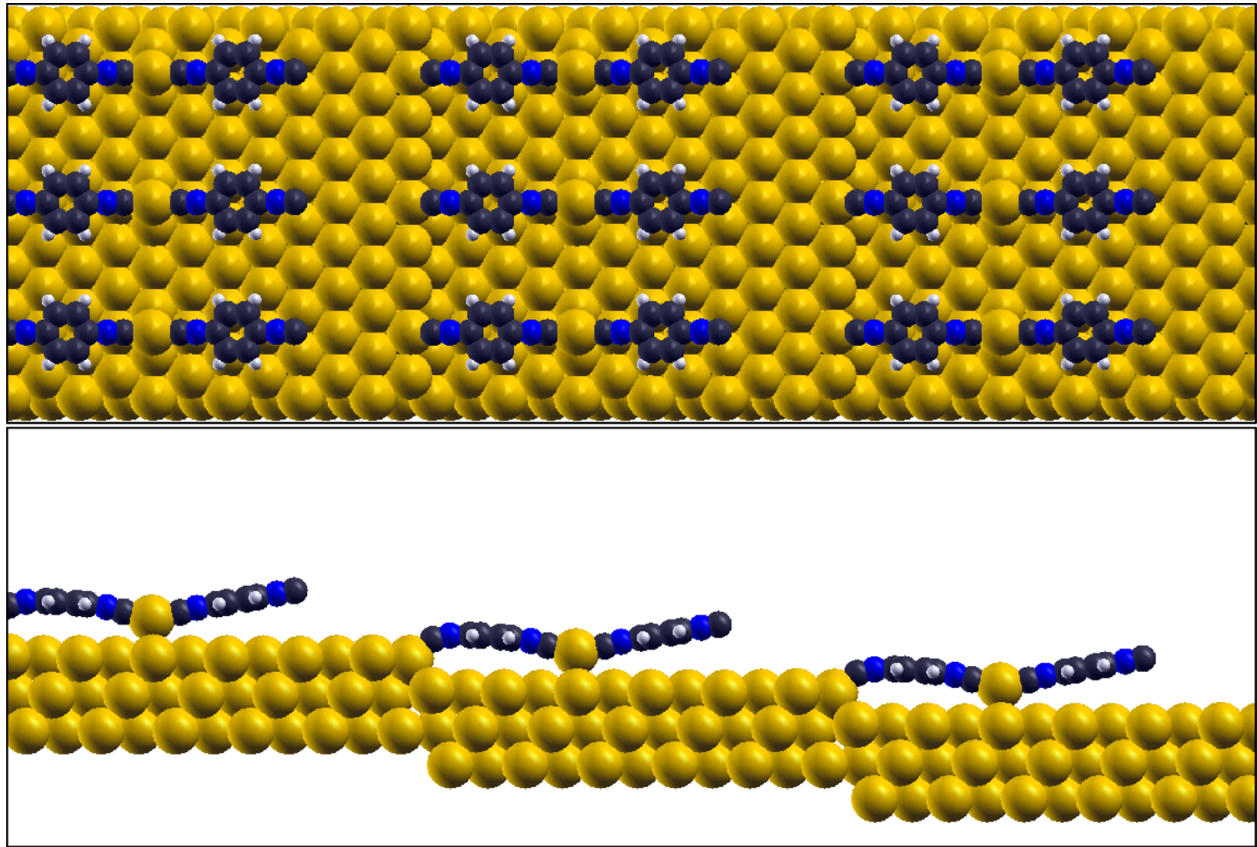

Figure 5. Final structure of a gold-PDI adatoms complex bound to an isocyanide molecule on a gold step edge.

that the PDI molecule on the step edge is now substantially tilted (at $\sim 30^{\circ}$ to the surface), with the plane of the aryl ring perpendicular to it. Clearly, such a tilted geometry will facilitate coupling between the isocyanide terminus and a gold atom in the adatom complex. To illustrate this, a gold adatom complex was placed at a 3-fold hollow site next to the tilted PDI molecule, which causes the adatom complex to tilt somewhat. The final structure, when the isocyanide has bonded to the gold adatom is shown in Figure 5. This is stable by $\sim 0.4 \mathrm{eV}(\sim 38$ $\mathrm{kJ} / \mathrm{mol}$ ) with respect to the initial state shown in Figure 4, so that the reaction is thermodynamically feasible. Interestingly, the PDI terminus is now even closer to the surface than when bound to a step edge, thereby facilitating further oligomerization by $\mathrm{Au}-\mathrm{PDI}$ adatom complex coupling. This trans effect is common in organometallic complexes ${ }^{26}$ and is consistent with the structure of isolated $\mathrm{Au}-\mathrm{PDI}$ chains. ${ }^{2}$ The energy profile for the transition from the initial (Figure 4) to the final (Figure 5) states is shown in Figure 6, along with along with selected structures along the reaction pathway. An animation of the motion is available in the Supporting Information section. As

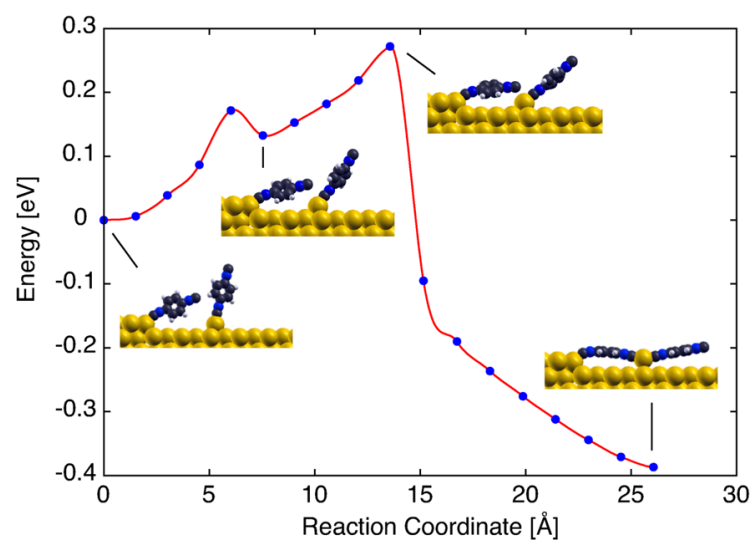

Figure 6. Energy profile for the binding of a gold adatom complex to a PDI molecule attached to a gold step edge. the reaction proceeds, both PDI molecules begin to tilt, continuing to do so until the isocyanide group forms a bond with the gold adatom. The gold adatom moves slightly to optimize the bonding to the isocyanide group. This is accompanied by addition tilting of the terminal PDI molecule, which then becomes oriented close to parallel to the surface. The energy profile shows an initial, metastable intermediate and a second, higher barrier when the gold-isocyanide bond is formed. The height of the second barrier is $\sim 0.28 \mathrm{eV}(\sim 27 \mathrm{~kJ} /$ $\mathrm{mol}$ ), indicating that this step is relatively rapid at room temperature.

Since van der Waals' interactions have been found to affect the energetics and, in some cases, the structure of adsorbates on gold, ${ }^{17,20,27,28}$ similar calculations were performed by including these interactions. The resulting initial structure (analogous to that shown in Figure 4) that includes van der Waals' interactions (displayed in Figure 7), clearly show that they have a profound effect on the initial-state structure. Now the PDI molecule at the step edge is parallel and much closer to the surface because of the van der Waals' interactions. This minimizes the initial interaction with the $\mathrm{Au}-\mathrm{PDI}$ adatom complex, which now has a structure that is closer to the isolated species (Figure 2). The structure of the final state is similarly affected (Figure 8), where the PDI molecule at the terminus of the growing oligomer is also parallel and close to the surface. The oligomerization reaction is now significantly more exothermic (by $\sim 1.2 \mathrm{eV}, \sim 115 \mathrm{~kJ} / \mathrm{mol}$ ). The corresponding energy profile calculated by including van der Waals interactions is shown in Figure 9. Now, in spite of the increased exothermicity, the barrier height is only very minimally affected, but is still relatively low, $\sim 0.3 \mathrm{eV}(\sim 29$ $\mathrm{kJ} / \mathrm{mol}$ ). This is consistent with the structural evolution, indicating that the reaction occurs with a relatively early transitions state. ${ }^{29-33}$

These results suggest that PDI oligomerizes on the surface by a mobile $\mathrm{Au}-\mathrm{PDI}$ adatom complex approaching a tilted, bound PDI molecule, either on a step edge or at the end of a growing -(Au-PDI - oligomer chain, to form a gold-isocyanide bond. 


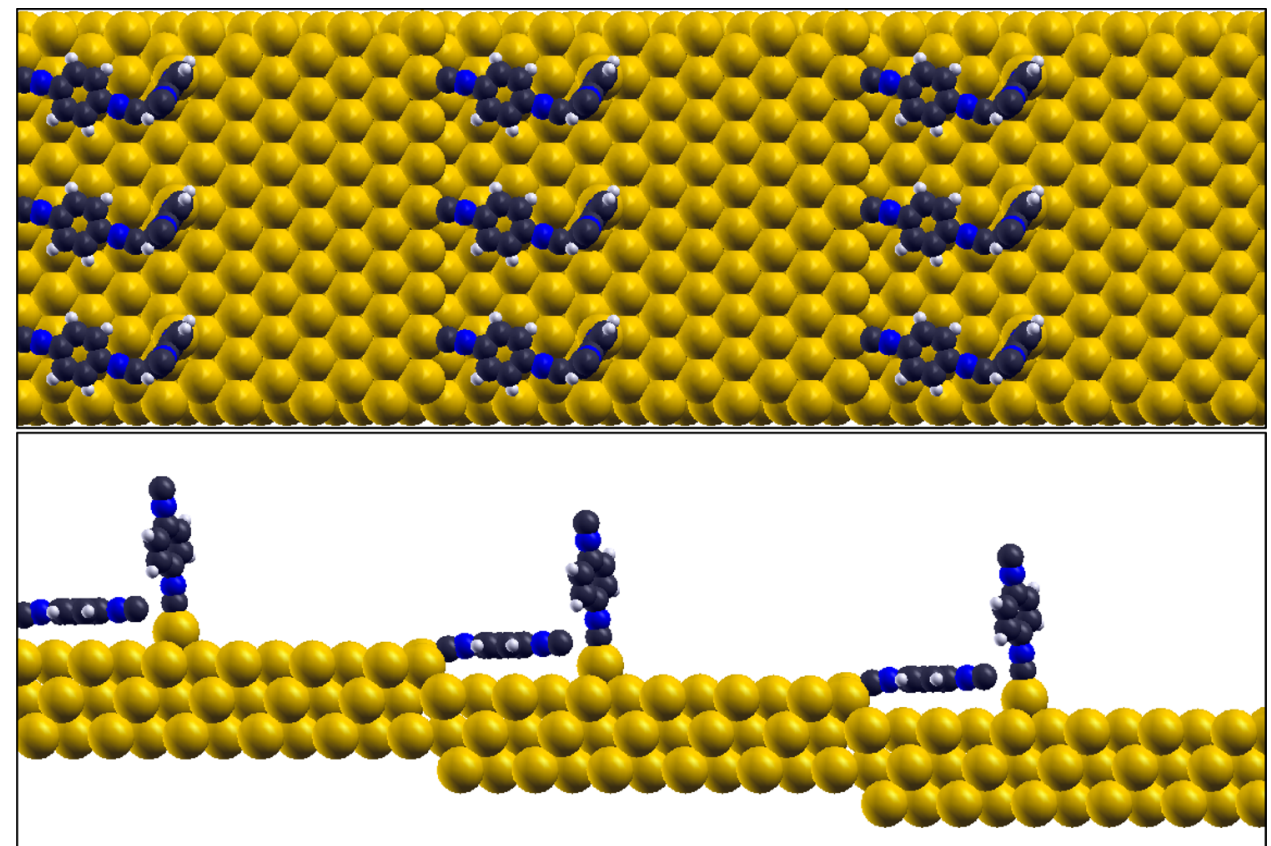

Figure 7. Calculated structure of a PDI molecule bound to a gold step, with a second PDI molecule placed close to it and an initial configuration for calculating the reaction barrier, now including van der Waals' interactions.

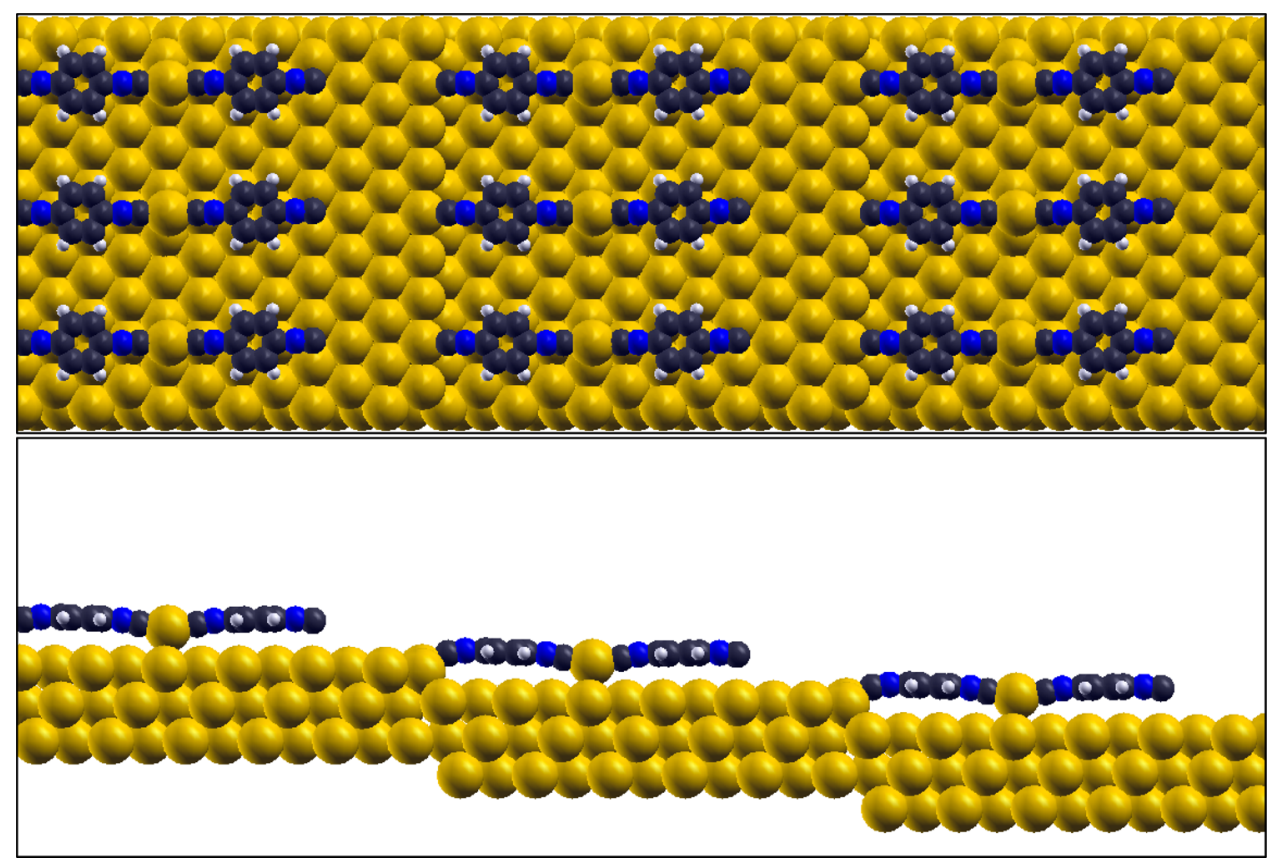

Figure 8. Final structure of a gold-PDI adatoms complex bound to an isocyanide molecule on a gold step edge, now including van der Waals' interactions.

However, rapid diffusion of the gold-adatom complex, while facilitating access to the end of a growing chain, may not reside for a sufficient time for the bond to form. To explore this, the energy of a gold-adatom complex was calculated as a function of its distance from a chain terminus. In this case, the goldPDI complex was placed at a particular site, and then the system was allowed to relax to its minimum energy, and the calculations were performed using van der Waals' corrections. The results are displayed in Figure 10 where the energies are referenced to that of a $\mathrm{Au}-\mathrm{PDI}$ adatom complex placed adjacent to the isocyanide terminus (as shown for the initial state in Figure 7). There are two possible sites for the PDIadatom complex one site away from the terminus (labeled Step 1 ), both of which are $\sim 50 \mathrm{meV}$ more stable than the closest position. However, the sites at two positions away (Step 2) are $\sim 100$ meV less stable than the closest site and the site at three steps away is less stable by $\sim 50 \mathrm{meV}$. This indicates that the $\mathrm{Au}-\mathrm{PDI}$ adatom complex can be trapped close to the terminus of the PDI molecule located at a step edge of growing Au-PDI chain, thereby accelerating the growth rate.

Finally, it has been observed that the Au-PDI oligomer chains, once formed on the surface are relatively mobile, ${ }^{1,2}$ 


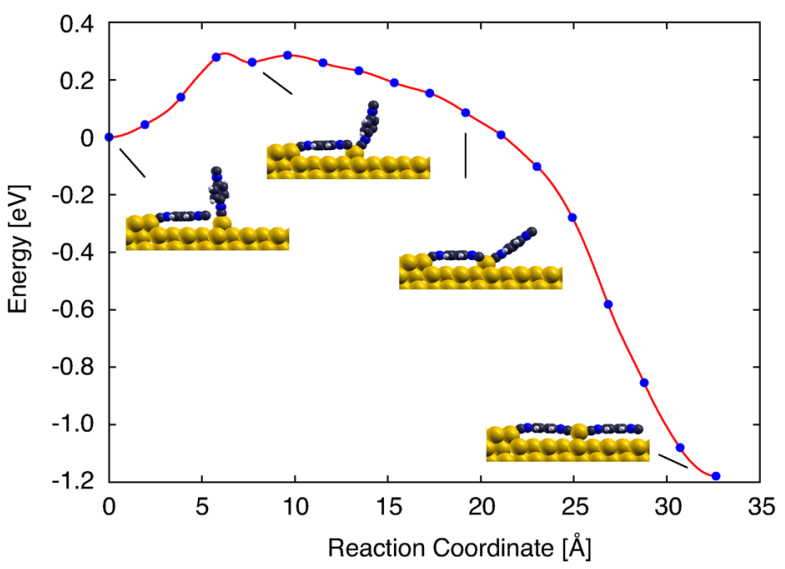

Figure 9. Energy profile for the binding of a gold adatom complex to a PDI molecule attached to a gold step edge, now including van der Waals' interactions.

which presumably assists in their ability to link between gold nanoparticles. ${ }^{9}$ In order to explore this effect, DFT calculations were performed to calculate the chain diffusion barrier and the results are displayed in Figure 11. This reveals a very low diffusion barrier of $\sim 1 \mathrm{~kJ} / \mathrm{mol}$ per repeat unit. It is expected that the diffusion barrier will increase approximately linearly with the length of the oligomer chain in accord with the mobility of even quite long chains seen in STM.

The DFT calculations described above provide evidence for a plausible - (Au-PDI) - oligomerization pathway found following PDI adsorption on $\mathrm{Au}(111)$ and linking between gold nanoparticles. ${ }^{1,2}$ The propagating monomer is proposed to comprise a vertical, mobile $\mathrm{Au}-\mathrm{PDI}$ adatom complex. They repel each other on the surface, so do not tend to agglomerate but are attracted toward the terminus of a growing - $(\mathrm{Au}-$ PDI) - chain or a PDI molecule coordinated to a step edge. They can bind to the isocyanide group of the terminal PDI with a relatively low activation barrier $(\sim 28 \mathrm{~kJ} / \mathrm{mol})$ thus rapidly
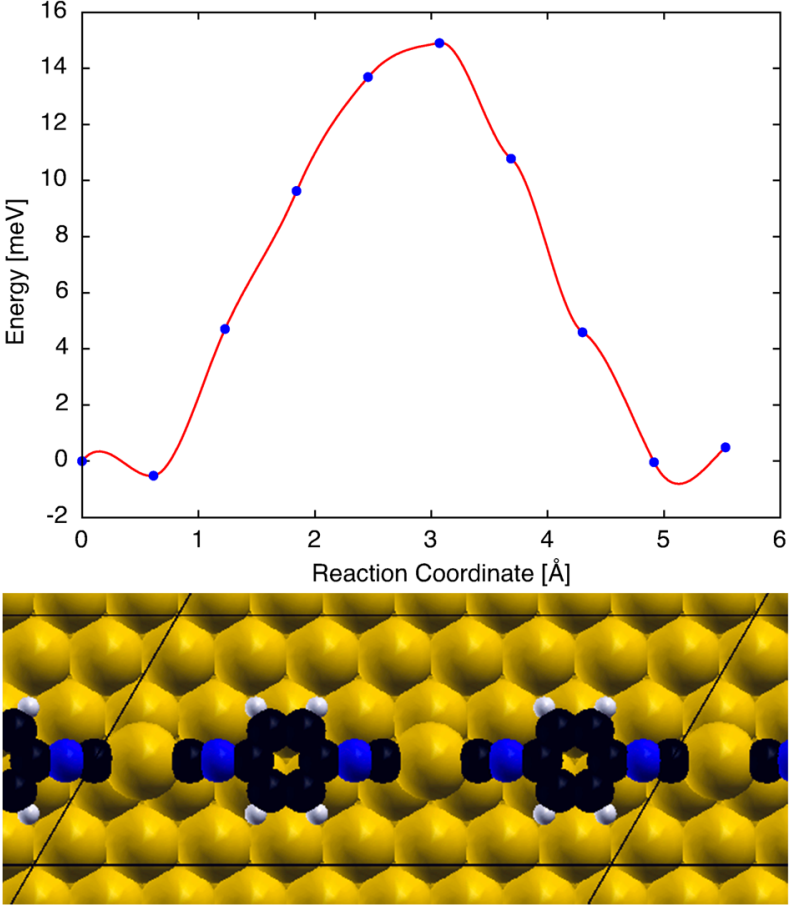

Figure 11. (a) The calculated activation barrier for the diffusion of an $\mathrm{Au}-\mathrm{PDI}$ oligomer chain from one sites to an adjacent on $\mathrm{Au}(111)$. (b) A depiction of the initial and final state of the Au-PDI oligomer chains.

propagating the chain. It is anticipated that such an oligomerization pathway is available to any bifunctional molecule that can bond strongly to gold to form a mobile adatom complex. This pathway requires that the second, uncoordinated group be sterically isolated from the surface to prevent it forming $\eta^{2}$ - complexes that would preclude propagation. This condition is clearly fulfilled in 1,4-phenylene diisocyanide, where the isocyanide groups are located para to

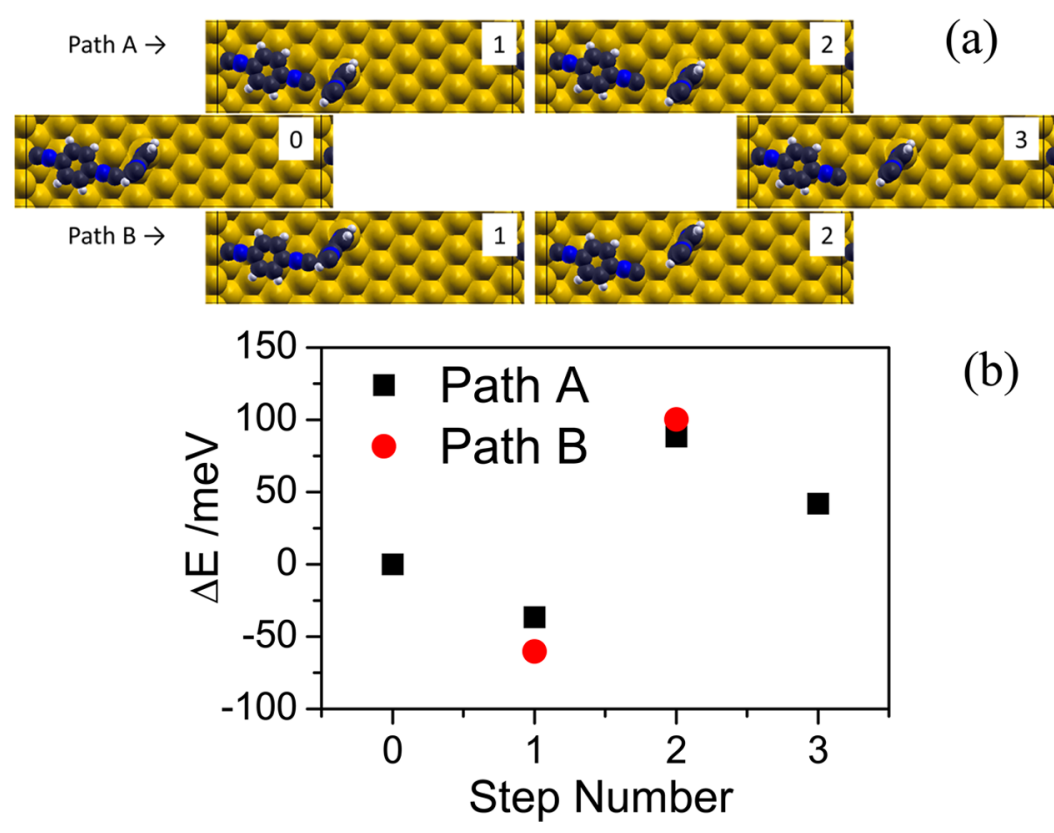

Figure 10. Plot of the energy of the gold adatom complex as a function of its distance from the growing isocyanide terminus. Indicated also are the structures used for each calculation. 
each other, and where the molecule is rigid. Thiols also form thiolates on gold by binding to gold adatoms ${ }^{4,5,7}$ so that it is anticipated that dithiols would form similar oligomeric chains as long as the second thiolate was constrained so as not to bind to the surface. It is therefore expected that this lateral selfassembly pathway should be available to a range of bifunctionalized molecules, as long as the molecular structure prevents the second functional group from accessing the surface.

The tilt of the isocyanide molecule observed in the transition state suggests possible strategies for obtaining a preferential molecular orientation of the growing chain if the molecularadatom complex is polar. In this case, the application of an electric field parallel to the surface while dosing to bridge between gold nanoparticles would be expected to result in an oligomer with a preferential orientation with respect to the imposed electric field. While this could not be achieved with the nanoparticle arrays on mica that have been used to explore the electron transport properties of bridged nanoparticles because of the low potential difference between adjacent nanoparticles, ${ }^{9}$ it should be possible for systems with discrete nanogaps. The effect of an external force on the rate of a chemical reaction has been modeled using Bell theory ${ }^{34-36}$ where the reaction rate constant under the influence of an external force is give by

$$
k(F)=k_{0} \exp \left(\frac{\vec{F} \cdot \overrightarrow{\Delta x^{\ddagger}}}{k_{\mathrm{B}} T}\right)
$$

Here $k_{0}$ is the thermal rate constant, $\vec{F}$ is the external force exerted on the molecule and $\overrightarrow{\Delta x^{*}}$ is a vector along the reactant to the transition state. In the case of PDI on gold, reaction from the initial to the transition state involves a tilting of the coordinating PDI molecule as the molecule on the step edge forms a bond with the gold. Since the reaction coordinate in this case involves a molecular tilt, a torque $\tau$ exerted to rotate the molecule through an angle $\Delta \theta^{\ddagger}$ will lead to a modified equation:

$$
k(\tau)=k_{0} \exp \left(\frac{\tau \Delta \theta^{\ddagger}}{k_{\mathrm{B}} T}\right)
$$

In order to estimate the value of $\tau \Delta \theta^{\ddagger}$, it is assumed that a molecule analogous to PDI has a dipole moment $\mu$ and is similarly initially oriented perpendicular to the surface (Figure 7), and that oligomerization proceeds via a similar pathway as discussed above. The torque exerted on the polar molecule will depend on the alignment of the dipole moment with respect to the field, where one alignment will lower the barrier, while the opposite assignment will increase it. For an electric field of magnitude $E$ parallel to the surface $\tau \Delta \theta^{\ddagger}= \pm \mu E \cos \left(\Delta \theta^{\ddagger}\right)$, where the + and - indicate the orientations of the dipole moment of the polar molecule. Note that this enhancement will be identical irrespective of the electrode at which the oligomerization is initiated. Thus

$$
k_{ \pm}(\tau)=k_{0} \exp \left(\frac{ \pm \mu E \cos \left(\Delta \theta^{\ddagger}\right)}{k_{\mathrm{B}} T}\right)
$$

leading to $k_{+}(E, \mu) / k_{-}(E, \mu)=\exp \left(\left(2 \mu E\left(\cos \left(\Delta \theta^{\ddagger}\right)\right) /\left(k_{\mathrm{B}} T\right)\right)\right.$. The relative propagation rates will lead to a preferential orientation in one direction that is in direct proportion to their rate constants. The resulting values of $N_{+}(E, \mu) / N_{-}(E, \mu)=$ $\exp \left(\left(2 \mu E\left(\cos \left(\Delta \theta^{\ddagger}\right)\right) /\left(k_{\mathrm{B}} T\right)\right)\right.$ are estimated by assuming that the $\Delta \theta^{\ddagger}$ is similar to the value of $\sim 30^{\circ}$ seen for the tilt from the initial to the transition state in Figure 8. The values of orientational excess, calculated from

$$
\begin{aligned}
& \left(\left(N_{+}(E, \mu)-\left(N_{-}(E, \mu)\right) /\left(\left(N_{+}(E, \mu)+\left(N_{-}(E, \mu)\right)\right.\right.\right.\right. \\
& \quad=\left(\frac{N_{+}(E, \mu)}{N_{-}(E, \mu)}-1\right) /\left(\frac{N_{+}(E, \mu)}{N_{-}(E, \mu)}+1\right)
\end{aligned}
$$

are estimated for electric fields between $10^{7}$ and $10^{9} \mathrm{~V} / \mathrm{m}$ for dipole moments between 0.5 and $3 \mathrm{D}$ at $T=300 \mathrm{~K}$. In this case, completely random orientations yield a value of zero, while prefect alignment results in a value of unity. The results are plotted versus electric field in Figure 12. This reveals that

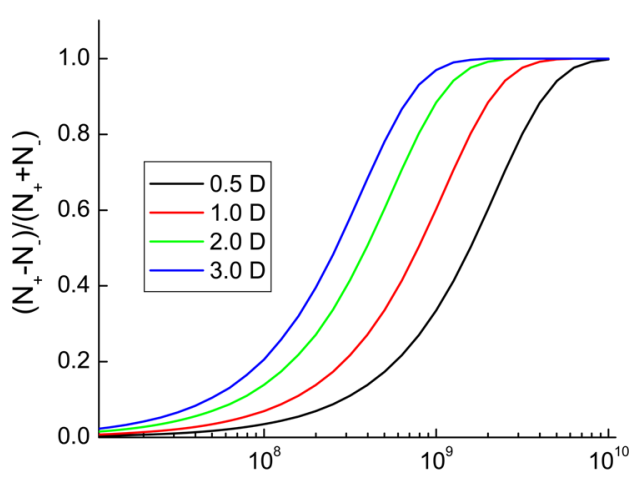

Electric Field/V/m

Figure 12. Plot of the orientational excess, $\left(N_{+}-N_{-}\right) /\left(N_{+}+N_{-}\right)$ versus electric field in $\mathrm{V} / \mathrm{m}$ for molecules with dipole moments of 0.5 , 1,2 , and $3 \mathrm{D}$.

significant orientation occurs with applied electric fields of $\sim 10^{8}$ $\mathrm{V} / \mathrm{m}$, and that almost complete alignment can occur if the fields reach $\sim 10^{9} \mathrm{~V} / \mathrm{m}$. Such fields can easily be attained by the application of $\sim 1 \mathrm{~V}$ across a $10 \mathrm{~nm}$ gap.

\section{CONCLUSIONS}

DFT calculations have been used to identify the self-assembly pathway for the formation of - (Au-PDI) - oligomers from the adsorption of 1,4-phenylene diisocyanide (PDI) on a $\mathrm{Au}(111)$ at room temperature. The propagating monomer is proposed to consist of a vertical Au-PDI adatom complex, which is relatively mobile on the $\mathrm{Au}(111)$ surface. The adatom complexes tend to repel each other on the surface, thereby preventing them from agglomerating. It is found that the goldPDI adatom complex is attracted to the isocyanide terminus of a growing oligomer chain or to an isocyanide group bonded to a step edge, where it bonds such that the molecular plane is oriented close to parallel to the surface, due to van der Waals' interactions with the substrate. The oligomer propagation step occurs by the gold adatom of the adatom complex coupling with the isocyanide terminus, followed by a tilting of the PDI molecule. This creates a geometry which allows this oligomerization cycle to repeat.

Based on this model, the possibility of aligning polar molecules that are analogous to PDI in an external electric field is explored. It is found that an external field of $\sim 10^{8} \mathrm{~V} / \mathrm{m}$ causes significant preferential molecular orientation, while, with an external field of $\sim 10^{9} \mathrm{~V} / \mathrm{m}$, almost complete molecular 
alignment is found. This suggests a possible strategy for forming molecular diodes.

\section{ASSOCIATED CONTENT}

\section{S Supporting Information}

Videos 1, 2, and 3. This material is available free of charge via the Internet at http://pubs.acs.org.

\section{AUTHOR INFORMATION}

\section{Corresponding Author}

*Phone: (414) 229-5222; FAX: (414) 229-5036; E-mail: wtt@ uwm.edu.

\section{Notes}

The authors declare no competing financial interest.

\section{ACKNOWLEDGMENTS}

RA acknowledges support of this work by the Jordanian government. We thank Professor Werner Hofer for assistance with simulating STM images using bSKAN.

\section{REFERENCES}

(1) Boscoboinik, J.; Kestell, J.; Garvey, M.; Weinert, M.; Tysoe, W. Creation of Low-Coordination Gold Sites on $\mathrm{Au}(111)$ Surface by 1,4Phenylene Diisocyanide Adsorption. Top. Catal. 2011, 54, 20-25.

(2) Boscoboinik, J. A.; Calaza, F. C.; Habeeb, Z.; Bennett, D. W.; Stacchiola, D. J.; Purino, M. A.; Tysoe, W. T. One-Dimensional Supramolecular Surface Structures: 1,4-Diisocyanobenzene on $\mathrm{Au}(111)$ Surfaces. Phys. Chem. Chem. Phys. 2010, 12, 11624-11629.

(3) Zhou, J.; Acharya, D.; Camillone, N.; Sutter, P.; White, M. G. Adsorption Structures and Electronic Properties of 1,4-Phenylene Diisocyanide on the $\mathrm{Au}(111)$ Surface. J. Phys. Chem. C 2011, 115, 21151-21160.

(4) Maksymovych, P.; Sorescu, D. C.; Yates, J. T. Gold-AdatomMediated Bonding in Self-Assembled Short-Chain Alkanethiolate Species on the $\mathrm{Au}(111)$ Surface. Phys. Rev. Lett. 2006, 97, 146103.

(5) Maksymovych, P.; Yates, J. T. Au Adatoms in Self-Assembly of Benzenethiol on the $\mathrm{Au}(111)$ Surface. J. Am. Chem. Soc. 2008, 130, 7518-7519.

(6) Lennartz, M. C.; Atodiresei, N.; Müller-Meskamp, L.; Karthäuser, S.; Waser, R.; Blügel, S. Cu-Adatom-Mediated Bonding in ClosePacked Benzoate/Cu(110)-Systems. Langmuir 2008, 25, 856-864.

(7) Voznyy, O.; Dubowski, J. J.; Yates, J. T.; Maksymovych, P. The Role of Gold Adatoms and Stereochemistry in Self-Assembly of Methylthiolate on $\mathrm{Au}(111)$. J. Am. Chem. Soc. 2009, 131, 1298912993.

(8) Kestell, J.; Abuflaha, R.; Boscoboinik, J.; Garvey, M.; Bennett, D. W.; Tysoe, W. Determination of Adsorbate Structures from 1,4Phenylene Diisocyanide on Gold. J. Phys. Chem. Lett. 2014, submitted for publication.

(9) Kestell, J.; Abuflaha, R.; Boscoboinik, J. A.; Bai, Y.; Bennett, D. W.; Tysoe, W. T. Linking Gold Nanoparticles with Conductive 1,4Phenylene Diisocyanide-Gold Oligomers. Chem. Commun. 2013, 49, $1422-1424$.

(10) Aviram, A.; Ratner, M. A. Molecular Rectifiers. Chem. Phys. Lett. 1974, 29, 277-283.

(11) Elbing, M.; Ochs, R.; Koentopp, M.; Fischer, M.; von Hänisch, C.; Weigend, F.; Evers, F.; Weber, H. B.; Mayor, M. A Single-Molecule Diode. Proc. Natl. Acad. Sci. U. S. A. 2005, 102, 8815-8820.

(12) Lörtscher, E.; Gotsmann, B.; Lee, Y.; Yu, L.; Rettner, C.; Riel, H. Transport Properties of a Single-Molecule Diode. ACS Nano 2012, 6, 4931-4939.

(13) Batra, A.; Darancet, P.; Chen, Q.; Meisner, J. S.; Widawsky, J. R.; Neaton, J. B.; Nuckolls, C.; Venkataraman, L. Tuning Rectification in Single-Molecular Diodes. Nano Lett. 2013, 13, 6233-6237.
(14) Kresse, G.; Joubert, D. From Ultrasoft Pseudopotentials to the Projector Augmented-Wave Method. Phys. Rev. B 1999, 59, 17581775.

(15) Kresse, G.; Hafner, J. Ab Initio Molecular Dynamics for Liquid Metals. Phys. Rev. B 1993, 47, 558-561.

(16) Perdew, J. P.; Burke, K.; Ernzerhof, M. Generalized Gradient Approximation Made Simple. Phys. Rev. Lett. 1996, 77, 3865-3868.

(17) Ramalho, J. P. P.; Gomes, J. R. B.; Illas, F. Accounting for van der Waals Interactions Between Adsorbates and Surfaces in Density Functional Theory Based Calculations: Selected Examples. RSC Adv. 2013, 3, 13085-13100.

(18) Sohn, Y.; Pradhan, D.; Zhao, L.; Leung, K. T. Anomalous Oxidation Resistance of "Core-Only" Copper Nanoparticles Electrochemically Grown on Gold Nanoislands Prefunctionalized by 1,4Phenylene Diisocyanide. Electrochem. Solid State 2012, 15, K35-K39.

(19) Liu, W.; Carrasco, J.; Santra, B.; Michaelides, A.; Scheffler, M.; Tkatchenko, A. Benzene Adsorbed on Metals: Concerted Effect of Covalency and van der Waals Bonding. Phys. Rev. B 2012, 86, 245405.

(20) Mura, M.; Gulans, A.; Thonhauser, T.; Kantorovich, L. Role of van der Waals Interaction in Forming Molecule-Metal Junctions: Flat Organic Molecules on the $\mathrm{Au}(111)$ Surface. Phys. Chem. Chem. Phys. 2010, 12, 4759-4767.

(21) Kelkkanen, A. K.; Lundqvist, B. I.; Nørskov, J. K. van der Waals Effect in Weak Adsorption Affecting Trends in Adsorption, Reactivity, and the View of Substrate Nobility. Phys. Rev. B 2011, 83, 113401.

(22) Duy, L.; Maral, A.; Adam, K.; Talat, S. R. The Role of van der Waals Interaction in the Tilted Binding of Amine Molecules to the $\mathrm{Au}(111)$ Surface. J. Phys: Condens. Mater. 2012, 24, 222001.

(23) Tkatchenko, A.; Scheffler, M. Accurate Molecular van der Waals Interactions from Ground-State Electron Density and Free-Atom Reference Data. Phys. Rev. Lett. 2009, 102, 073005.

(24) Henkelman, G.; Uberuaga, B. P.; Jonsson, H. A Climbing Image Nudged Elastic Band Method for Finding Saddle Points and Minimum Energy Paths. J. Chem. Phys. 2000, 113, 9901-9904.

(25) Li, Y.; Lu, D.; Swanson, S. A.; Scott, J. C.; Galli, G. Microscopic Characterization of the Interface between Aromatic Isocyanides and $\mathrm{Au}(111)$ : A First-Principles Investigation. J. Phys. Chem. C 2008, 112, 6413-6421.

(26) Coe, B. J.; Glenwright, S. J. Trans-Effects in Octahedral Transition Metal Complexes. Coordin. Chem. Rev. 2000, 203, 5-80.

(27) Chwee, T. S.; Sullivan, M. B. Adsorption Studies of $\mathrm{C}_{6} \mathrm{H}_{6}$ on $\mathrm{Cu}$ (111), Ag (111), and Au (111) within Dispersion Corrected Density Functional Theory. J. Chem. Phys. 2012, 137, 134703.

(28) Lüder, J.; Sanyal, B.; Eriksson, O.; Puglia, C.; Brena, B. Comparison of van der Waals Corrected and Sparse-Matter Density Functionals for the Metal-Free Phthalocyanine/Gold Interface. Phys. Rev. B 2014, 89, 045416.

(29) van Santen, R. A.; Neurock, M. Molecular Heterogeneous Catalysis: A Conceptual and Computational Approach; Wiley-VCH: Weinheim/Cambridge, 2006.

(30) Evans, M. G.; Polanyi, M. Inertia and Driving Force of Chemical Reactions. Trans. Farad. Soc. 1938, 34, 11-24.

(31) Logadottir, A.; Rod, T. H.; Nørskov, J. K.; Hammer, B.; Dahl, S.; Jacobsen, C. J. H. The Brønsted-Evans-Polanyi Relation and the Volcano Plot for Ammonia Synthesis over Transition Metal Catalysts. J. Catal. 2001, 197, 229-231.

(32) Santen, R. A. v.; Neurock, M.; Shetty, S. G. Reactivity Theory of Transition-Metal Surfaces: A Brønsted-Evans-Polanyi Linear Activation Energy-Free-Energy Analysis. Chem. Rev. 2009, 110, 20052048.

(33) Cushing, G. W.; Navin, J. K.; Donald, S. B.; Valadez, L.; Johánek, V.; Harrison, I. C-H Bond Activation of Light Alkanes on Pt(111): Dissociative Sticking Coefficients, Evans-Polanyi Relation, and Gas-Surface Energy Transfer. J. Phys. Chem. C 2010, 114, 1722217232.

(34) Kauzmann, W.; Eyring, H. The Viscous Flow of Large Molecules. J. Am. Chem. Soc. 1940, 62, 3113-3125.

(35) Bell, G. Models for the Specific Adhesion of Cells to Cells. Science 1978, 200, 618-627. 
(36) Ribas-Arino, J.; Marx, D. Covalent Mechanochemistry: Theoretical Concepts and Computational Tools with Applications to Molecular Nanomechanics. Chem. Rev. 2012, 112, 5412-5487. 